\title{
Comunicación para la democracia: jóvenes y movimientos sociales en la era digital
}

\author{
Communication for democracy: \\ young people and social movements in the digital age
}

Amaro La Rosa Pinedo

Universidad Femenina del Sagrado Corazón

\section{INTRODUCCIÓN}

El compromiso cívico es un instrumento esencial para impulsar el desarrollo de la sociedad y fortalecer la democracia en el mundo contemporáneo. En esta perspectiva, como recursos de comunicación e interacción de creciente impacto global, los medios sociales son ahora vehículos muy importantes para promover la conducta cívica, intercambiar opiniones, motivar la discusión en la comunidad, desarrollar las actitudes de los ciudadanos e impulsar las acciones colectivas que son imperativas para la construcción y el reforzamiento de los ideales y prácticas democráticas.

Según la Unión Internacional de Telecomunicaciones (1), 2 mil 700 millones de personas, es decir alrededor del $40 \%$ de la población global, están conectadas a Internet y los 6 mil 800 millones de usuarios de teléfonos móviles están muy cerca de alcanzar al total mundial de población. Evidentemente, en la práctica estas no son simples estadísticas, denotan la existencia de un gran volumen de recursos tecnológicos en manos de la sociedad civil y de la humanidad en su conjunto, que pueden utilizarlos en su actividad cotidiana.

La naturaleza interactiva y la inmediatez de los avanzados recursos de la Web 2.0 permiten la puesta en marcha de múltiples y crecientes conexiones entre los seres humanos y promueven la activa participación social de los individuos, grupos y comunidades, más allá de las fronteras, inclusive desafiando las restricciones al uso de Internet que intentan imponer los regímenes autoritarios.

No somos los primeros en utilizar, en una interpretación propia, los términos de Umberto Eco (2), para aseverar que ante la evidencia del desarrollo tecnológico que suponen Internet y los medios sociales, pueden adoptarse dos modalidades de comportamiento. Por un lado estarían los apocalípticos o modernos ludditas - a decir de aquellos grupos de trabajadores que en los inicios de la Revolución Industrial se dedicaban a destruir

1 Licenciado en Psicología, especializado en Psicología de la Comunicación, con estudios de posgrado en CIESPAL y en la Universidad de Chile; docente e investigador en el Programa de Comunicación de la Universidad Femenina del Sagrado Corazón (UNIFÉ) Lima. 
maquinarias-, quienes se resisten a su uso y continúan empleando recursos obsoletos; y por otro lado, los integrados, quienes adoptan entusiastamente las innovaciones e inclusive motivan a los demás para que las utilicen. Desde luego, los apocalípticos de hoy tienden a ser cada vez menos y pueden convertirse en los integrados a ultranza del mañana, pues tarde o temprano, deberán emplear los recursos tecnológicos a los que potencialmente tienen acceso, los cuales conllevan una serie de ventajas: facilitan las tareas cotidianas, perfeccionan los productos informativos, permiten mayores estándares de competitividad y amplían los horizontes del individuo.

El presente artículo realiza una breve apreciación sobre el involucramiento cívico de los jóvenes en vinculación con su uso de las redes o medios sociales, cuya tasa de uso es mayor en comparación con otras edades. Nos interesa conocer cómo los nativos digitales incrementan su participación cívica activa en los movimientos sociales. Intentamos hacer la descripción, el análisis y la interpretación del referido tema partiendo de la evidencia científica más reciente, los hechos de la realidad y los aportes teóricos de diversas disciplinas. Se propone un modelo tomando en cuenta la necesidad de la construcción de conocimientos científicos en el ámbito estudiado.

\section{Web 2.0 y medios sociales}

Es indudable que con la comunicación mediada por computadoras que supone Internet, han aparecido nuevas modalidades de intercambio comunicativo, convertidas en indispensables.

Ampliando un criterio previamente establecido (3), mencionamos algunos aspectos de la Web 2.0 que la hacen muy asequible, facilitando la creación de contenidos por parte de los usuarios:

- Simplicidad en el uso, pues quien es usuario de una red social se encuentra con un software muy amigable que le permite manejarse con mucha sencillez.
- No requiere aprenderse el HTML ni ningún otro lenguaje complejo, tal como el de las primeras generaciones de computadoras.

- Placer de utilización: El usuario puede pasarse hora tras hora conectado e inmerso en el mundo de la red social que le interesa, dada la naturaleza de los contenidos que encuentra, que se incrementan constantemente y a los que puede ingresar y agregar los datos que le resulten convenientes o apropiados. Al respecto, un estudio empírico de 389 usuarios de Facebook encontró que el uso de medios sociales está determinado por normas internas del individuo así como por su identidad social que supone el desarrollo de actitudes (4).

- Separación de forma y contenido: Se acercan mucho ambos, pues es el usuario quien aporta el volumen más importante de contenido, por cierto que sobre la base de un software preestablecido, de uso gratuito, el cual ni siquiera requiere instalarse y que más aún recibe los aportes de sus usuarios, quienes como verdaderos desarrolladores, producen utilitarios y aplicaciones que amplían los horizontes de la red social. Tal es el caso de Facebook, que ha incrementado el interés de los usuarios por haber incorporado un sinnúmero de recursos útiles, interesantes o motivadores desarrollados por sus usuarios. Por este rasgo, Bruns (5) prefiere denominarlos "produsuarios", por cuanto sus prácticas comunicativas contribuyen al mejoramiento de los contenidos mediante su construcción y ampliación.

Dada la convergencia de las telecomunicaciones y la digitalización, el desarrollo de los teléfonos inteligentes ha posibilitado el acceso a Internet en general y a los medios sociales en particular.

Palen (6) plantea que la conectividad producida por los teléfonos móviles involucra la conexión social de múltiples aspectos de la vida de los individuos a los cuales la autora describe con casos concretos como 


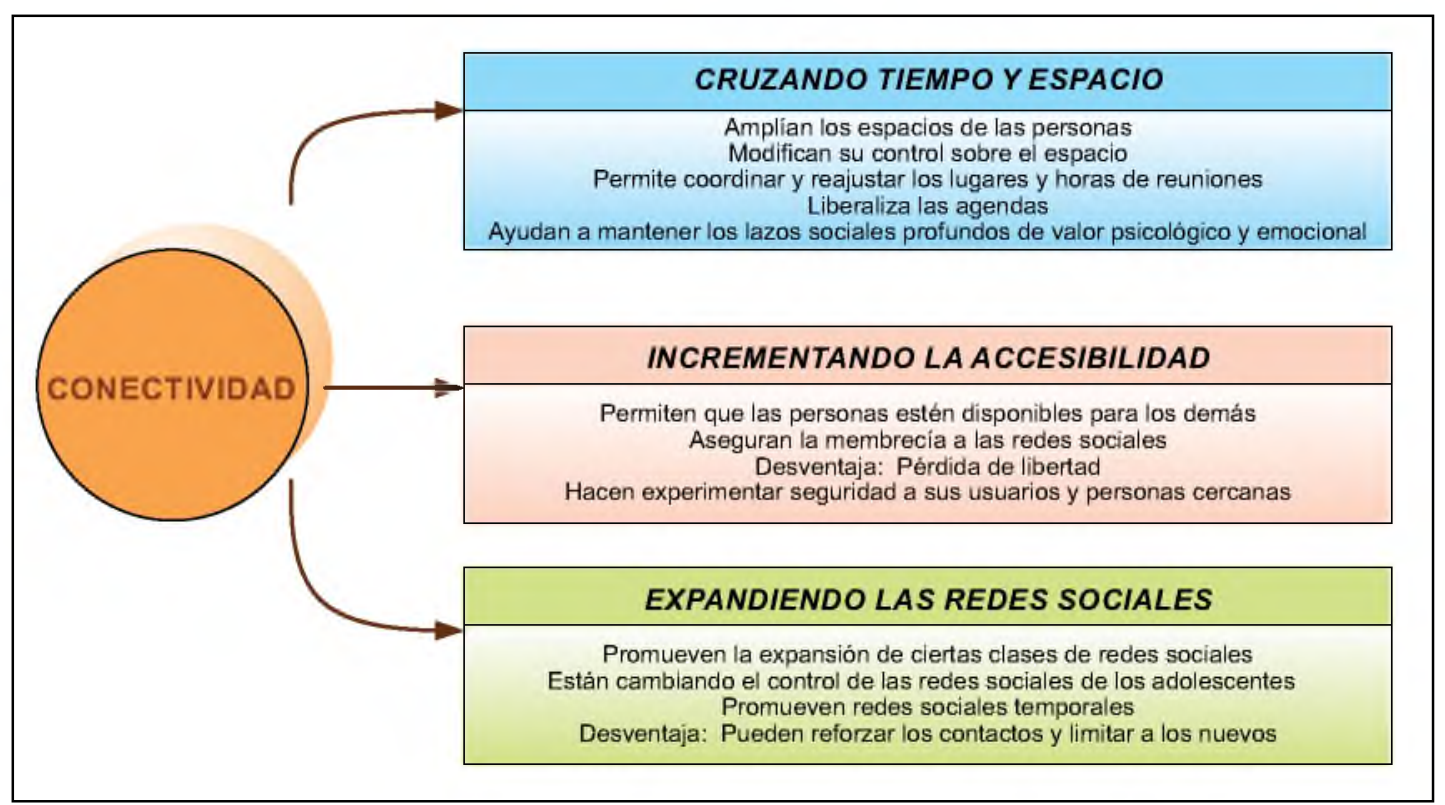

Figura $N^{\circ} 1$ : La conectividad de los teléfonos móviles, basada en Palen (2002).

el cruce del espacio y tiempo, el incremento de la accesibilidad y la expansión de las redes sociales (figura 1). A partir de este criterio podemos visualizar algunas de las razones por las cuales los teléfonos móviles son recursos invalorables para la activa participación en los movimientos sociales.

De acuerdo con este punto de vista, estimamos que los teléfonos incrementan la potencialidad informativa de las redes sociales al asociarse con su movilidad, ampliando los espacios de los individuos. Igualmente, permiten reajustar los parámetros para las reuniones y promueven redes sociales temporales.

\section{Movimientos sociales en la era digital}

Harlow (7) sostiene que existen dos criterios de aproximación para explicar el papel de Internet en los movimientos sociales:

- Internet como facilitador del activismo tradicional offline, que refuerza con recursos propios las herramientas que ya poseen los activistas.

- Internet como generador de nuevas modalidades de activismo y resistencia.
Hoy los movimientos sociales cuentan con mayores recursos para su difusión y motivación a la acción, inclusive en situaciones de restricción de las libertades para la sociedad civil. Sobre este particular, Faris (8) considera que los medios sociales contribuyen a la superación del silencio que se impone a los medios masivos en los regímenes dictatoriales posibilitando un fluido flujo de comunicación, indispensable para las acciones colectivas.

La sociedad civil encuentra en los medios sociales una serie de ventajas (9):

- Comunicación en tiempo real.

- Transmisión instantánea de mensajes a miles de personas.

- Cobertura ilimitada.

- Posibilidades de acción colectiva a pesar de las restricciones oficiales.

- Rápida reacción colectiva frente a los hechos.

- Posibilidades de cambiar a último momento los lugares de encuentro.

- Gran potencialidad para lograr apoyo 


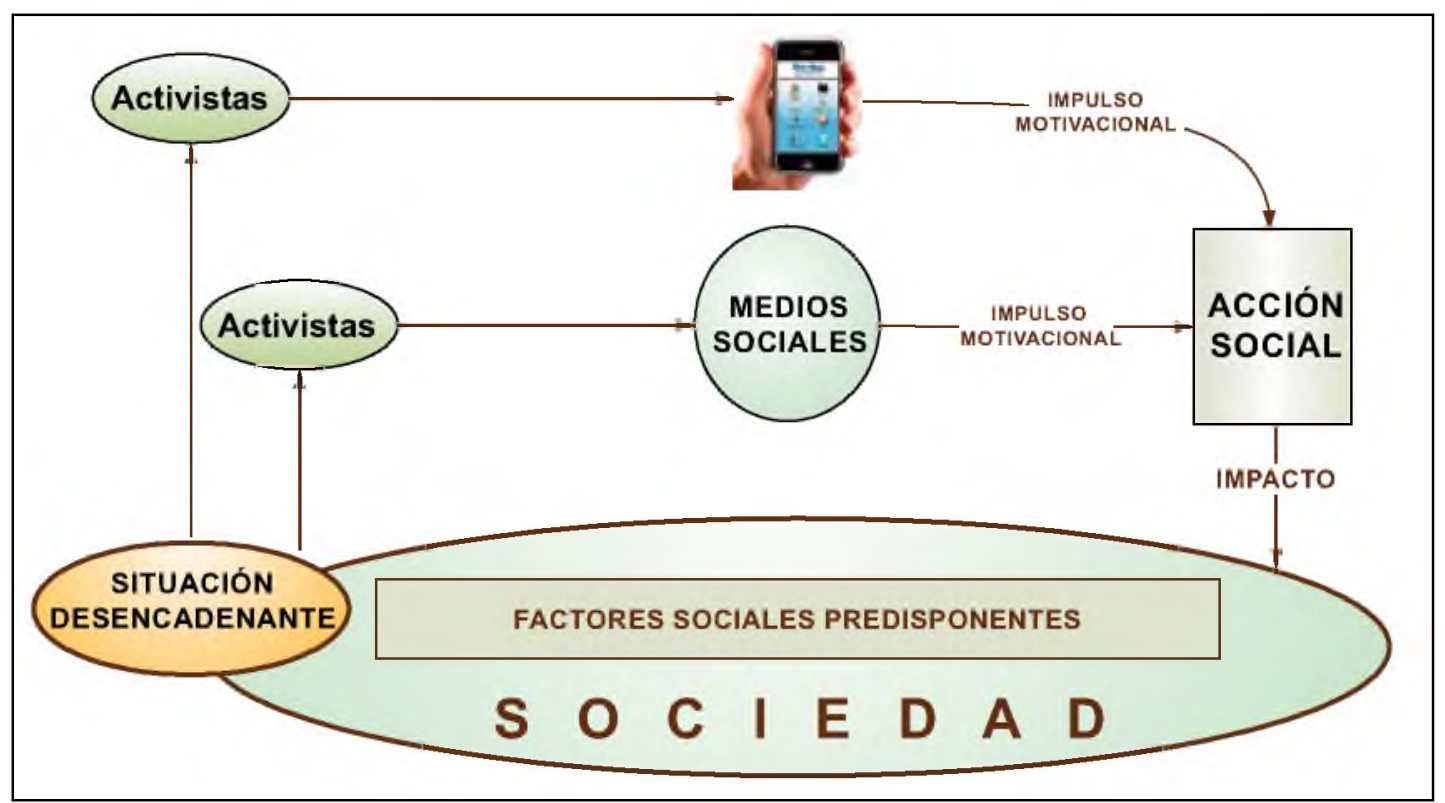

Figura $\mathrm{N}^{\circ}$ 2: Los movimientos sociales en la era digital (elaboración propia).

internacional porque sus mensajes cruzan las fronteras de los países.

- Limitada inversión en recursos tecnológicos para las organizaciones de la sociedad civil.

- Posibilidad de subir inmediatamente información a bases de datos internacionales y redes internacionales de medios.

- Posibilidades de compartir experiencias con otras organizaciones a costo cero.

En cuanto a los teléfonos móviles, el aporte de los rasgos de su conectividad, el incremento de la calidad de su cámara fotográfica y de su videocámara hace posible contar con registros de suma utilidad para los movimientos sociales.

Luego de un análisis de la problemática y partiendo de una visión transdisciplinaria, consideramos que los movimientos sociales en la era digital poseen los siguientes rasgos fundamentales:

- Uso intensivo de medios digitales: Emplean medios sociales tales como Twitter, Facebook, Youtube. Asimismo utilizan los teléfonos móviles inteligentes con acceso a Internet.

- Transversalidad: Participación de diversos sectores sociales.

- Horizontalidad en la organización.

- Rapidez en la toma de decisiones.

- Rapidez en el paso de la motivación a la acción social.

- Desvinculación de intereses políticos partidarios.

Cuando hablamos de los movimientos sociales y la participación de los medios sociales, no queremos aseverar que basta solamente el accionar de tales medios y teléfonos móviles para que los movimientos sociales se activen rápidamente. Lim (10) afirma, por ejemplo, que la denominada Primavera Árabe no surgió espontáneamente, pues fue la resultante de años de movimientos de la sociedad civil que se hicieron efectivos tanto online como offline. Sobre este particular creemos necesario plantear un modelo que esperamos validar más adelante, basándonos en la evidencia científica así como es su contrastación con la realidad. 
Según la figura 2, el esquema de acción de los movimientos sociales en la era digital se da de la siguiente manera: a) los movimientos sociales se hacen efectivos en una determinada sociedad en la cual existen ciertos factores predisponentes; b) ante una situación desencadenante, activistas que tienen acceso a redes sociales y a teléfonos móviles, difunden su criterio; y c) el impulso motivacional genera una acción social que produce a su vez un impacto de mayor o menor trascendencia sobre la sociedad.

\section{Jóvenes y movimientos sociales}

López (11) sostiene que los jóvenes activistas realizan diversos usos de Internet:

- Información: Internet es fuente de información sobre los movimientos sociales y sus agendas. En función del desarrollo de Internet se modifica el tradicional circuito de la información.

- Comunicación: Internet como recurso para participar en el debate público.

- Coordinación: Factor clave que incluye: a) coordinación informativa: para reforzar el conocimiento y la participación; b) coordinación propositiva: para promover la acción; c) coordinación cibersolidaria: para apoyo en campañas específicas; y d) coordinación reactiva: de carácter defensivo.

Los ciberactivistas que actúan en la promoción y gestión de movimientos sociales van incorporando paulatinamente los nuevos recursos digitales que se ponen a disposición de los usuarios. Si hacemos un mapeo de los movimientos sociales de mayor trascendencia en los últimos años, encontramos que en estos grupos son los jóvenes quienes tienen una participación activa y parten frecuentemente de situaciones concretas que afectan a su sociedad. En su análisis de los movimientos sociales del siglo XXI, Voss y Williams (12) anotan que el énfasis actual es en la construcción de organizaciones capaces de democratizar la sociedad civil a partir de sus propias problemáticas.
En una diversidad de casos queda evidenciado que las redes sociales son en la época contemporánea un recurso de comunicación de primera importancia. Son especialmente los jóvenes quienes las utilizan, involucrándose de manera activa en la promoción de los cambios sociales que su sociedad necesita. La reconocida revista Correo de la Unesco le dedica su edición de julio-setiembre de 2011 al protagonismo de los jóvenes en el cambio social y lo hace con el título de edición "Cómo los jóvenes cambian el mundo".

Esta participación de los jóvenes en los movimientos sociales de la era 2.0, según nuestro criterio, se explica por varias razones:

- Su dominio de los recursos y aplicaciones de Internet y de los teléfonos móviles inteligentes con acceso a Internet que le posibilitan la creación y envío de información actualizada mediante diversas plataformas.

- Su gran capacidad de convocatoria a través de las redes que han establecido, que están en posibilidad de crecer y que les permiten movilizar en tiempo reducido un numeroso grupo de personas y lograr un considerable impacto.

- Su grado de identificación con los movimientos sociales. El estudio de Tindall y Bates (13) revela que los jóvenes tienden identificarse más con los movimientos sociales que los adultos, pese a los costos que ello implique.

- El interés de los jóvenes por participar en el cambio social. A pesar de las afirmaciones y evidencias de una diminución en sus motivaciones por la participación hemos observado en diversos países procesos muy interesantes de empoderamiento digital. La activa participación política de jóvenes utilizando medios sociales en procesos electorales y movimientos sociales son dos clases de hechos que caracterizan en la actualidad su acción política, cuyos importantes casos destacamos en el 
presente artículo.

- La independencia de los medios sociales de las tradicionales modalidades de comunicación, en tanto las audiencias juveniles pueden ser al mismo tiempo receptores y productores de mensajes sobre los hechos sociales, convirtiéndose en líderes de opinión. En este sentido, la formación de la opinión pública y su propia naturaleza han experimentado profundas modificaciones, dependiendo en algunos casos de un hashtag, un tweet o de un video viral colgado en Youtube.

Revisemos algunos de los movimientos sociales de los últimos años, en los cuales los jóvenes tuvieron un papel fundamental.

El escenario es la Plaza Tahrir en El Cairo. En medio de un clima de tensión social, luego del derrocamiento del presidente de Túnez, miles de personas se agrupan en este lugar céntrico. Han sido convocadas casi silenciosamente mediante los diversos recursos que permiten Internet y vehiculizan los modernos teléfonos inteligentes. En un tiempo relativamente corto lograron algo que parecía imposible: el 11 de febrero de 2011 el presidente Hosni Mubarak renunciaba al cargo y todo hace indicar que pasará el resto de su vida en prisión.

En 2006, usando diversos recursos de Internet, especialmente los fotologs y mensajería instantánea, los escolares chilenos convocaron a movilizaciones para exigir soluciones a problemas del transporte y la educación. A decir de Valderrama (14), lograron la participación activa de más de 600 mil estudiantes de todo el país en lo que se conoció como "huelga virtual" y "revolución de los pingüinos", provocando una crisis en el gobierno.

\#Yo Soy 132 es un movimiento social mexicano de jóvenes quienes plantean la democratización de los medios, empleando como recursos a los medios sociales para desarrollar acciones de protesta. En su tesis, Elizabeth Crumpacker (15) indica que su meta es combatir la desigual distribución del poder y cuestionar el sistema de medios de comunicación existente. Señala que, aunque parezca paradójico, solo publica dos a tres posts en su cuenta de Facebook, sin embargo estos reciben miles de "me gusta" (la autora registra 220, 326). Ante la elección como presidente de Peña Nieto, representante del $\mathrm{PRI}$, realizó diversas acciones offline para motivar a los jóvenes a favor del movimiento.

Las protestas en las calles de algunos grupos de jóvenes en Lima surgieron como reacción luego de los acuerdos en el Congreso del Perú para elegir a los miembros del Tribunal Constitucional y al Defensor del Pueblo. Usando redes sociales y teléfonos móviles convocaron a protestar en contra de lo que denominaron la repartija. El resultado fue que el Parlamento dio marcha atrás en su decisión.

\section{CONCLUSIONES}

Los jóvenes, como nativos digitales, son los principales usuarios de Internet y a decir de lo observado, en lo que va del siglo $X X \mid$, tienen una participación activa en la génesis de los movimientos sociales, que comparando con los de épocas previas de la historia, ya no se basan en reivindicaciones de largo plazo ni enarbolan una bandera partidaria. Hoy plantean la pronta solución de problemáticas globales o locales con plena independencia de los poderes tradicionales.

Los medios sociales tales como Twitter, Facebook, Youtube y Flickr, entre otros, se han convertido en recursos invalorables para la movilización de los jóvenes hacia la acción social en pro de la democracia en diversos lugares del mundo. Los teléfonos inteligentes han permitido registrar los hechos vinculados con los movimientos sociales y llevar al mundo las imágenes y los videos testimoniales de los reclamos de los jóvenes así como las reacciones de los gobiernos y sus representantes; aun en contra de las restricciones impuestas al acceso a Internet.

Haciendo una analogía con los términos del 
argot de Internet y de los medios sociales, las acciones y respuestas de los movimientos sociales en los que participan los jóvenes están en permanente construcción y sus integrantes interactúan en tiempo real con la meta de cambiar al menos una parte de los chips de la historia.

\section{REFERENCIAS BIBLIOGRÁFICAS}

1. ITU. The world in 2013: ICT facts and figures [Internet]. 2012. Disponible en: http://www.itu.int/en/ITU-D/ Statistics/Documents/facts/ ICTFactsFigures2013-s.pdf

2. Eco, U. Apocalípticos e integrados. España: DeBOLSILLO; 2004.

3. La Rosa A. Redes sociales en la web, comunicación y comportamiento social. Avances en Psicología, UNIFÉ. 2010; 17 (1): 85-95.

4. Cheunga $C$, Leeb M. A theoretical model of intentional social action in online social networks. Decision Support Systems. 2010; 49 (1): 24-30.

5. Bruns A. From prosumption to produsage. In Towse R, Handke C (eds.). Handbook on the Digital Creative Economy. Gloucestershire: Edward Elgar; 2013. p. 67-78.

6. Palen L. Mobile telephony in a connected life. Communications of the Acm. 2002; 45 (3): 78-82.

7. Harlow S. Social media and social movements: Facebook and an online Guatemalan justice movement that moved offline. New Media \& Society. 2012; 14(2): 225-243.

8. Faris D. La révolte en réseau: le "printemps árabe" et les médias sociaux. Politique étrangère. $2012 ; 1$ : 99-109.

9. La Rosa A, Roumate F. Social media, new paradigm for civil society: Cases United States of America and Latin America. Presented at the The 9th Annual Latin America and Caribbean Regional Conference of the International Society for Third Sector Research. Santiago de Chile, 2013.
10. Lim M. Clicks, Cabs, and Coffee Houses: Social Media and Oppositional Movements in Egypt, 2004-2011. Journal of Communication. 2012; 62: 231-248.

11. López S. Jóvenes, Internet y movimiento antiglobalización: usos activistas de las nuevas tecnologías. Revista Estudios de Juventud. 2007; 76: 183-199

12. Voss K, Williams M. The local in the global: Rethinking social movements in the new millennium. Institute for Research on Labor and Employment University of California, Berkeley. 2009. Disponible en:

http://repositories.cdlib.org/iir/iirwps/ iirwps-177-09

13. Tindall D, Bates K. Youth activism and old trees: A study of youth participation in the Vancouver Island Wilderness Preservation Movement. Presented at the American Sociological Association 93rd Annual Meeting. San Francisco, 1998.

14. Valderrama L. B. Jóvenes, ciudadanía y tecnologías de Información y comunicación. El movimiento estudiantil chileno. Revista Latinoamericana de Ciencias Sociales, Niñez y Juventud. 2013; 11(1): 123-135.

15. Crumpacker E. \#Yo Soy 132 and Occupy: Social Movements and the Media. BA Thesis. Scripps College Claremont. 2013 